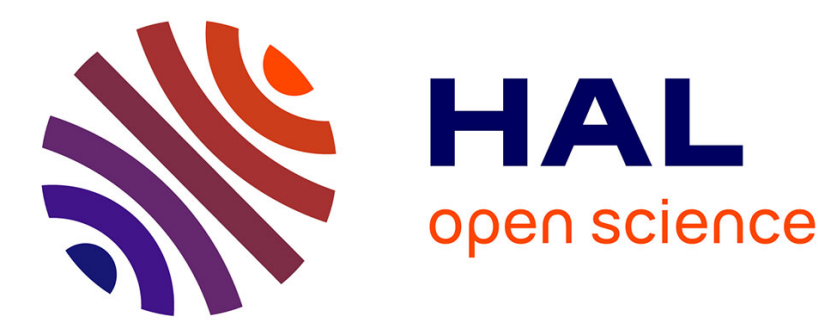

\title{
Influence of reservoir size on the adsorption path in an ideal pore
}

Joël Puibasset, Edouard Kierlik, Gilles Tarjus

\section{To cite this version:}

Joël Puibasset, Edouard Kierlik, Gilles Tarjus. Influence of reservoir size on the adsorption path in an ideal pore. Journal of Chemical Physics, 2009. hal-02110078

\section{HAL Id: hal-02110078 \\ https://hal.science/hal-02110078}

Submitted on 25 Apr 2019

HAL is a multi-disciplinary open access archive for the deposit and dissemination of scientific research documents, whether they are published or not. The documents may come from teaching and research institutions in France or abroad, or from public or private research centers.
L'archive ouverte pluridisciplinaire HAL, est destinée au dépôt et à la diffusion de documents scientifiques de niveau recherche, publiés ou non, émanant des établissements d'enseignement et de recherche français ou étrangers, des laboratoires publics ou privés. 


\title{
Influence of reservoir size on the adsorption path in an ideal pore
}

\author{
Joël Puibasset* \\ Centre de Recherche sur la Matière Divisée, CNRS-Université d'Orléans, \\ $1 b$ rue de la Férollerie, 45071 Orléans Cedex 02, France \\ Edouard Kierlik ${ }^{\dagger}$ and Gilles Tarjus ${ }^{\ddagger}$ \\ LPTMC, CNRS-UMR 7600, Université Pierre et Marie Curie, \\ boîte 121, $4 \mathrm{Pl}$. Jussieu, 75252 Paris Cedex 05, France
}

(Dated: July 13, 2009)

\begin{abstract}
We consider the influence of the relative size of the gas reservoir on the states visited by a simple fluid adsorbed in a nanopore of ideal geometry (a slit). We focus on the intermediate states that appear in between the main hysteresis branches comprising gaslike and liquidlike states and we study the adsorption and desorption paths actually followed by the system as one changes the reservoir size. We find that these paths may display discontinuous sections associated with transitions between different nonuniform states. We also discuss the stability of the states in such situations.
\end{abstract}




\section{INTRODUCTION}

The sequence of intermediate states visited by a fluid during the course of its adsorption or desorption in a porous material has attracted wide interest over the years, both for theoretical and practical reasons. The question is especially arduous when adsorption/desorption in a grand-canonical experimental setup, which usually features hysteretic behavior, proceeds through a phase transition associated with capillary condensation (or evaporation): then, the fluid density jumps from a gaslike to a liquidlike value (or the reverse) at constant chemical potential and the associated sequence of configurations through which the fluid passes during the process corresponds to out-of-equilibrium states. It has been suggested however that information on the out-of-equilibrium process can be obtained by considering adsorption under canonical-ensemble conditions that could constrain the allowed fluid states. ${ }^{1}$ Some of the unstable states associated with the out-of-equilibrium capillary condensation or evaporation in the grand-canonical set-up (open system) could therefore be stabilized and their thermodynamic properties studied. ${ }^{1-7}$

More generally, it is known that the states among which a fluid evolves during capillary condensation or evaporation in a nano- or mesoporous solid may depend on the characteristics of the experimental setup. ${ }^{1-7}$ Specifically, they may be affected by the size of the gas reservoir in contact with the porous sample. A very large reservoir compared with the sample size corresponds to a grand-canonical ensemble for the adsorbed fluid, whose chemical potential is externally controlled. A vanishingly small reservoir on the other hand leads to a "petit"-canonical ensemble for the adsorbed fluid in which it is the adsorbed amount that is controlled. In between, one has a realization of a mixed statistical ensemble in which the total amount of fluid in the reservoir and the porous sample is fixed whereas the two subsystems, reservoir and sample, are at chemical equilibrium. The issue we address in this article is that of the nature of the "intermediate" states found inside the main (grand-canonical) hysteresis loop observed for adsorption isotherms when an ensemble intermediate between the "petit" - and grand-canonical situation is considered.

In a previous paper, ${ }^{7}$ we have investigated the effect of the relative size of the gas reservoir on adsorption of a fluid in inhomogeneous porous media. We have shown that the fluid passes through a sequence of states that are metastable in the grand-canonical ensemble, i.e. stable against small perturbations yet generically not with the lowest thermodynamic grand 
potential. In these systems, metastability is induced by the intrinsic inhomogeneity forced on the fluid configurations by the porous solid (and of course by the practical limitation on accessible observation times, which does not allow the system to escape from states in which it is trapped). In addition, we have found that the path among metastable states on adsorption or desorption depends on the reservoir size.

In the present work, we come back to an a priori simpler problem, that of adsorption in a pore of ideal geometry. Contrary to our previous study, there is no intrinsic inhomogeneity induced by the solid, at least in the (lateral) directions in which the ideal pore is uniform. If intermediate states are present in addition to the (laterally) uniform gaslike and liquidlike branches seen in grand-canonical adsorption, what are their origin, nature and sequence ? Are they metastable, as in an inhomogeneous mesoporous material, or "conditionally stable", 8 i.e. only stabilized by the constraint coming from the finite size of the reservoir ? If they are conditionally stable, could they be relevant to more complex geometries as well $?$

Answering these questions has experimental, theoretical and methodological interest:

- Experimental, as the physical reservoir in actual setups for fluid adsorption is of finite extent and its size may be comparable to that of the porous sample ${ }^{9}$; it is therefore worth exploring the consequences of such a situation in those cases where the porous solid can be reasonably well described as a collection of independent pores of ideal geometry (e.g., MCM41, activated carbons, etc).

- Theoretical, since the intermediate states stabilized with a finite reservoir may also be relevant for the out-of-equilibrium dynamics of the fluid in a grand-canonical situation (open system). Understanding the nature of the stability of these states and the possible "morphological transitions" 2 among those states would be valuable.

- Methodological: computer simulations are a tool to calculate in modeled systems thermodynamic quantities that may not be accessible in experiments. For an adsorbed fluid, thermodynamic integration of the adsorption isotherm is often used to compute the grand potential. The validity of the method, however, is questionable when discontinuities are present in the isotherm.

Although some of the above issues have already been discussed in the literature, most notably by Neimark and coworkers in a series of papers on the "gauge cell" method, ${ }^{3,4,10-13}$ we think it is worth providing additional insight from a specific angle: the effect of the 
relative size of the gas reservoir.

The paper is organized as follows. In section II, we introduce the setup and the model and describe both the simulation method and the thermodynamic quantities that we have computed. In section III we discuss our results on the influence of the relative size of the reservoir, which we have varied by more than two orders of magnitude, spanning representative situations from the grand-canonical ensemble to the canonical one. We conclude in section IV. Additional methodological aspects are presented in an appendix.

\section{SETUP, MODEL AND METHOD}

\section{A. Setup and model}

The experimental situation we want to model is that of isothermal adsorption in a porous sample which is in contact with a gas reservoir. One considers a starting situation where $N$ particles are placed inside two cells that are in thermal equilibrium with an infinite heat bath at temperature $T$. One of the cells represents a porous solid sample of volume $V_{P}$ and the other a reservoir of volume $V_{R}$. The total volume is then equal to $V=V_{P}+V_{R}$. We allow mass exchange between the cells, whose volumes are kept fixed, so that at equilibrium the chemical potential of particles inside the reservoir is equal to the chemical potential of particles inside the porous solid. This statistical ensemble is a special case of the Gibbs ensemble $^{14}$ where the two cells are not allowed to exchange volume.

We measure the average amount of particles $N_{P}$ present in the porous solid. Varying the total number of particles $N$ in small steps, one changes both the chemical potential of the cells $\mu(N)$, and the number of adsorbed particles $N_{P}(N)$. We then monitor the adsorption isotherm $\rho_{P}=N_{P} / V_{P}$ as a function of $\mu$. The limiting case in which $V_{R}$ is much larger than $V_{P}$ corresponds to the grand-canonical ensemble for the adsorbed fluid whereas $V_{R}=0$ corresponds to the canonical ensemble for the adsorbed fluid. Between these two extremes of the grand- and "petit"-canonical situation", one has an "intermediate canonical" ensemble, with a reservoir of tunable size compared to that of the sample. Following the terminology used by Neimark and coworkers in their gauge cell method, ${ }^{3,4,10-13}$ we refer to this ensemble as "mesoscopic canonical".

The system under investigation is a simple atomic fluid confined in a slit nanopore. The 
parameters of the model are more specifically chosen to mimic argon adsorbed in nanoporous solid carbon dioxide. ${ }^{15,16}$ The fluid-fluid interaction is described by a standard $(12-6)$ Lennard-Jones pair potential and all quantities are normalized to the Lennard-Jones parameters of the fluid, $\sigma$ (length) and $\epsilon$ (energy). The atomic roughness of the surface is neglected since it is not expected to strongly affect the various states that the adsorbed fluid can adopt in a simple geometry. The potential exerted by the solid on a fluid particle inside the pore is calculated by integrating a $12-6$ Lennard-Jones potential between fluid and wall particles over a uniform distribution of wall sites. The calculated reduced external potential is displayed in Fig. 1 as a function of the reduced distance to the wall. The width of the nanopore is 6 ( $x$ direction) and in the directions ( $y$ and $z$ ) parallel to the wall planes the sizes are 8 and 24 (see Fig. 1). Periodic boundary conditions are applied along the two directions $y$ and $z$ parallel to the planes. For convenience the fluid-fluid interactions are truncated at 3 atomic diameters.
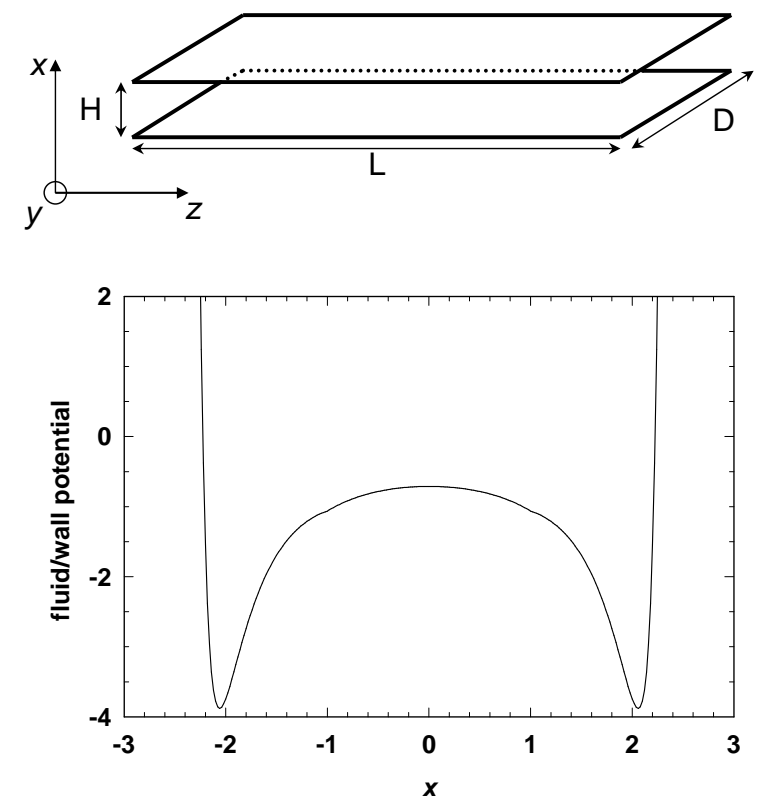

FIG. 1: Upper panel: Schematic representation of the slit pore with $H=6, D=8, L=24$ (units of Lennard-Jones $\sigma$ ). Lower panel: Fluid/wall external potential given as a function of the reduced distance to the wall (as obtained by integration over a uniform density of wall sites in the smooth-wall approximation).

This simple model already captures the main features to provide a description of the 
various states that the fluid can adopt in simple pores, when the relative size of the gas reservoir compared to that of the pore sample is varied.

\section{B. Simulation method}

The fluid adsorption properties are calculated by Monte-Carlo simulations. Thermalization of the adsorbed fluid is performed by particle-displacement trials whereas chemical equilibration between the adsorbed fluid and the reservoir is realized by particle-exchange trials. The acceptance probabilities are given by the Metropolis algorithm. The adsorption/desorption curves are determined as follows. The initial configuration is an empty pore. Few atoms are then added in one step to the system (pore plus reservoir). Actually, in order to mimic the experimental situation, the atoms are added to the reservoir cell first, and then allowed to relax by exchange and thermalization until the imbalance between the chemical potentials in the two cells (see below) goes to zero and chemical equilibrium is reached. $10^{6}$ Monte Carlo trials per particle are then performed to acquire statistics for computing averaged quantities. This gives the first point of the isotherm. The subsequent points are obtained according to the same procedure, i.e. a small increase in the number of atoms in the reservoir, followed by a relaxation run, and, finally, by a long run for acquisition. After complete adsorption has been achieved, the desorption isotherm can be calculated by decreasing the amount of particles in the reservoir step by step. (On the other hand, for an infinite reservoir, i.e. a grand canonical situation, it is the chemical potential which is increased or decreased stepwise.)

As shown below, the adsorption/desorption curves may present discontinuities (gaps or jumps). In order to improve the accuracy around these discontinuities, extra calculations are performed for intermediate points by adding or removing particles with smaller and smaller steps. The smallest possible increment is 1 atom, but in most cases such an extreme choice is not necessary, and steps of ten particles may be precise enough (more details are given below for some typical cases).

Note that the simulations being performed well below the critical point, the gas in the reservoir behaves to a very good approximation as an ideal gas (which we have checked by studying the first terms of the virial expansion). With the assumption of ideal behavior, the gas in the reservoir does not need to be explicitly treated, which speeds up the calculations. 


\section{Thermodynamic quantities in the mesoscopic canonical ensemble}

The whole (pore plus reservoir) system is supposed to be in equilibrium with a heat reservoir of infinite size and is thus in the canonical $(N, V, T)$ ensemble. Its thermodynamic properties are described by the usual Helmholtz free energy $F=-k T \ln Z(N, V, T)$ where $Z$ is the partition function of the whole (pore plus reservoir) system and $k$ the Boltzmann constant. The chemical potential $\mu$ is then defined as

$$
\mu=\left.\frac{\partial F(N, V, T)}{\partial N}\right|_{V, T}
$$

and it is the same everywhere in the system. Similarly, the thermodynamic pressure is defined as

$$
\Pi=-\left.\frac{\partial F(N, V, T)}{\partial V}\right|_{N, T},
$$

where the total volume is changed at constant ratio $V_{R} / V_{P}$.

On the other hand, the two subsystems (pore and reservoir) are in a mixed statistical ensemble. ${ }^{14,17,18}$ We introduce additional thermodynamic quantities for each subsystem. Their physical interpretation is intuitively clear but their rigorous foundation is not well established. First, and especially in a computer simulation, one of the subsystems may be of small size (despite the fact that the whole system is very large), which implies that fluctuations may not be negligible and that the knowledge of average quantities, such as the average number of atoms in the small subsystem, may not be sufficient. ${ }^{13}$ In our study, we do not encounter this problem since the two subsystems are always found to be big enough to ignore corrections due to fluctuations. Another more serious issue pertains to the meaning of the subsystems' thermodynamic quantities and the relationships among them when discontinuities occur in some of the quantities. For instance, in cases of interest, the canonical and grand-canonical ensembles for the confined fluid (corresponding to the two extreme limits of the mesoscopic canonical ensemble) may not be equivalent. More details on these points are given in Appendix. To remind the reader of these subtleties, we will add a tilde on all of these quantities.

One such quantity is the "chemical potential" of the gas in the reservoir which, by using the assumption that the gas is ideal and that the reservoir size is large enough for the gas behavior to be characterized by the average number of atoms $\left\langle N_{R}\right\rangle,{ }^{13}$ can be defined as

$$
e^{-\beta \widetilde{\mu}_{R}}=e^{-\beta \widetilde{\mu}_{R}^{i d}}=\frac{V_{R}}{\Lambda^{3}\left\langle N_{R}\right\rangle},
$$


where $\beta=1 / k T$ and $\Lambda$ is de Broglie wavelength. A "chemical potential" can also be defined and calculated for the fluid in the pore by the Widom test-particle equation: ${ }^{19,20}$

$$
e^{-\beta \widetilde{\mu}_{P}}=e^{-\beta\left(\widetilde{\mu}_{P}^{i d}+\widetilde{\mu}_{P}^{e x}\right)}=\frac{V_{P}}{\Lambda^{3}\left\langle N_{P}\right\rangle}\left\langle\frac{1}{V_{P}} \int_{V_{P}} d \mathbf{r} e^{-\beta W_{i n s}(\mathbf{r})}\right\rangle
$$

where the ideal part is defined as above from the average number of particles in the pore $\left\langle N_{P}\right\rangle$ (which is assumed to be sufficiently large), and the excess contribution is calculated with the usual Widom test-particle insertion algorithm: $W_{\text {ins }}(\mathbf{r})$ is the energy of interaction of the virtual particle (test particle), inserted at position $\mathbf{r}$ in the pore, with $N_{P}$ particles already adsorbed in the pore. This quantity is averaged over space to improve the efficiency of the algorithm. The brackets denote an average over atomic configurations (thermal fluctuations and particle exchanges). More details on these definitions are given in Appendix. It is also shown in this appendix that, at equilibrium, one has equality between these "chemical potentials", which both become equal to the conventional chemical potential introduced in Eq. (1):

$$
\widetilde{\mu}_{P}=\widetilde{\mu}_{R}=\mu
$$

this equality constitutes a criterion for the convergence of the simulation (which means that at least chemical equilibrium associated with exchange of particles between the two subsystems is achieved).

\section{EFFECT OF RESERVOIR SIZE}

\section{A. Grand-canonical isotherms}

Fig. 2 shows the adsorption/desorption isotherms and the grand potential in the slit pore obtained by Grand Canonical Monte Carlo (GCMC) simulation at a reduced temperature $k T / \epsilon=0.7$. One observes the expected hysteresis in mesopores, i.e. a gaslike branch corresponding to argon adsorbed at the walls, and a liquidlike branch corresponding to dense fluid filling the pore. The grand potential $\Omega_{P}$ is computed via the usual virtual volume variation method in the grand-canonical ensemble ${ }^{21,22}$ and is shown as symbols in the lower panel of Fig. 2. The grand potentials of the two branches intersect at coexistence. To determine more precisely the location of the coexistence point, we have also calculated the grand potential by thermodynamic integration of the adsorption isotherm, which provides 


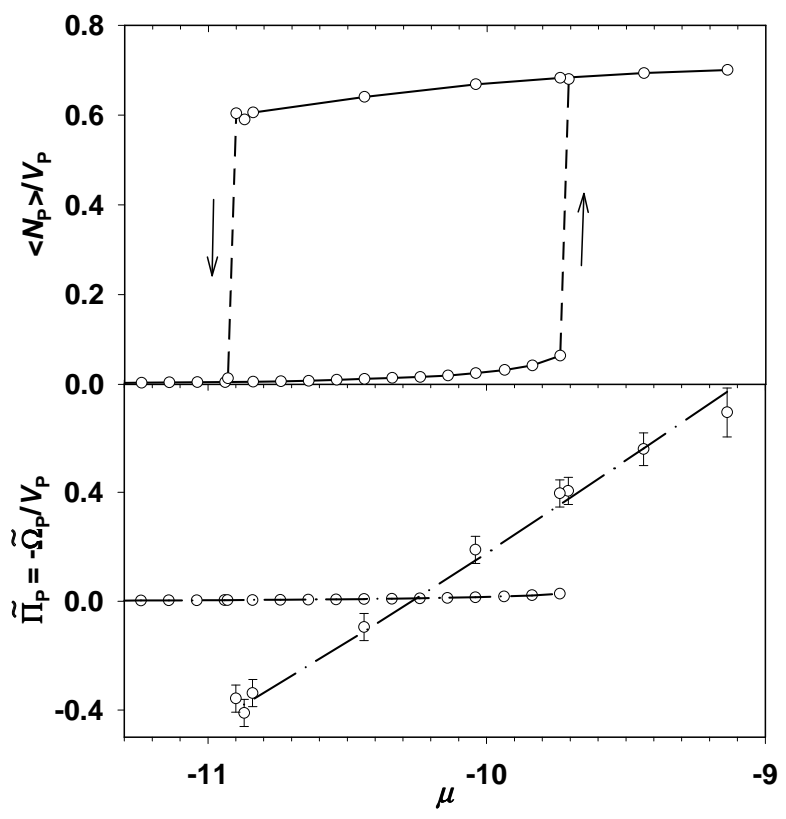

FIG. 2: GCMC adsorption/desorption isotherms (upper panel) and thermodynamic pressure (lower panel) for a Lennard-Jones fluid confined in the slit pore described in Fig. 1. Symbols: simulation points; solid lines and vertical dashed lines in the upper panel: guides to the eye showing gaslike and liquidlike branches as well as the (out-of-equilibrium) transitions from one branch to the other; dash doted line in the lower panel: fluid thermodynamic pressure obtained by thermodynamic integration of the adsorption isotherm (including a best-fit procedure, see text). The coexistence of the gaslike and liquidlike phases is located by the intersection of the grand-potential branches and occurs at $\mu_{\text {coex }} \simeq-10.25$.

continuous curves for the two branches. The gaslike branch is integrated from the lowchemical potential limit (ideal fluid). The liquidlike branch on the other hand is known up to a constant; the latter is determined by a best fit to the data obtained via the direct computation. (The point of highest density is discarded for the fit as its uncertainty is quite large because of a lack of convergence due to a low acceptance level of particle creation or removal at high density.) The results are shown as dash dotted lines in the lower panel of Fig. 2.

We stress that, because of the free-energy barriers that may trap the system in metastable states, the existence and the width of the hysteresis obtained in GCMC depends on the length of the simulation run, and on the efficiency of the Monte Carlo algorithm: hysteresis would 
disappear for infinitely long simulations. However, even in experiments in which accessible observation times are much longer than in simulations, a hysteresis is usually observed, whose properties are quite reproducible. In spite of the difference of typical time scales in experiments and in simulations, it is expected that the qualitative features and the origin of the hysteretic phenomenon are the same in both cases.

\section{B. Results in the mesoscopic canonical setting}

In GCMC, the reservoir is large enough to fix the chemical potential and provide or absorb the required number of particles during adsorption or desorption, in particular during the "out-of-equilibrium transitions" (jumps indicated by the vertical dashed lines in Fig. 2). On the other hand, for a finite reservoir-to-sample volume ratio, it is the total number of particles which is fixed, not the chemical potential. This has drastic consequences and allows one to explore possible fluid states inside the main GCMC hysteresis loop. Fig. 3 displays the adsorption/desorption isotherms obtained in the same slit pore and at the same temperature as previously for a reservoir of decreasing size, from $\sim 10^{3}$ down to less than 10 times the volume of the pore sample. The GCMC data are superimposed in each case

for comparison. Among the most notable changes, one finds: a reduction of the hysteresis effect between adsorption and desorption, the appearance of states of intermediate densities between the gaslike and liquidlike branches, and the persistence of discontinuites in the isotherms even when hysteresis vanishes.

The results obtained with a reservoir of volume 933 times the pore volume (Fig. 3a) are reminiscent of the GCMC results: on the time scale of the simulation, a hysteresis is observed and the gaslike and liquidlike branches obtained in the two cases are essentially superimposable within uncertainties. The most obvious difference with the GCMC appears in the transitions (jumps), which are not vertical anymore. To improve the accuracy around these jumps, we have reduced the increments in the total number of particles (many nearby points can be seen around the locations of the jumps and correspond to increments of \pm 10 atoms). Why are the jumps not vertical any more? The explanation goes as follows. Any large variation in the number of particles in the pore (during large jumps) induces the opposite variation in the reservoir (because of conservation of the total number of particles in the whole system). Due to the finite size of the reservoir, this implies a variation of the 

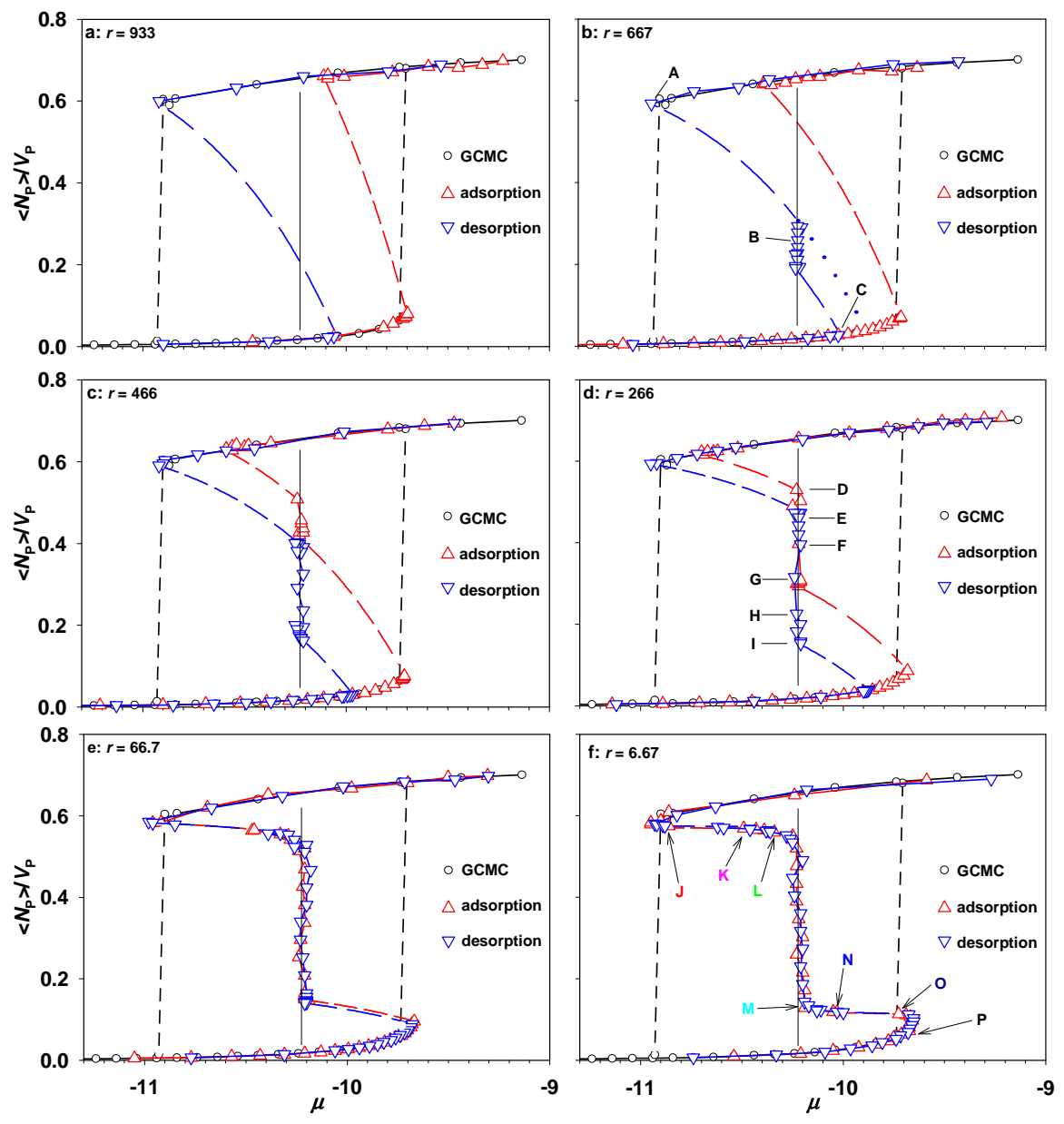

FIG. 3: Adsorption/desorption isotherms for a Lennard-Jones fluid in a slit pore obtained within the mesoscopic canonical ensemble for various reservoir-to-pore volume ratio $r$ given in the figure: from (a) to (f), $r$ decreases from 933 to 6.67. The GCMC results of Fig. 2 are superimposed in each panel (the thin vertical line corresponds to GCMC coexistence). The dashed lines (and the dotted line in (b)) represent the constraint relation in Eq. (6). The points labeled A to P are used in subsequent figures.

chemical potential in the reservoir (see section II-C). The constraint of fixed total number of particles $N$ (when the transition occurs) translates into a relation between the fluid density in the pore $\rho_{P}=\left\langle N_{P}\right\rangle / V_{P}$ and the fluid chemical potential $\mu$ :

$$
\rho_{P}(\mu)=r\left[\frac{N}{V_{R}}-\frac{e^{\beta \mu}}{\Lambda^{3}}\right]
$$

where we have introduced the volume ratio $r=V_{R} / V_{P}$. The corresponding curves are shown as dashed lines in the figure. These lines would be vertical straight lines in the grand- 
canonical ensemble $(r \rightarrow \infty)$ and horizontal straight lines in the canonical ensemble $(r=0)$. Eq. (6) allows one to express the change in adsorbed fluid density as a function of the change in the chemical potential between the starting point and the end point of the jumps. The good connection between these lines of constraint and the simulation points confirms that we have chosen increments in the total number of particles around the transition that are small enough.

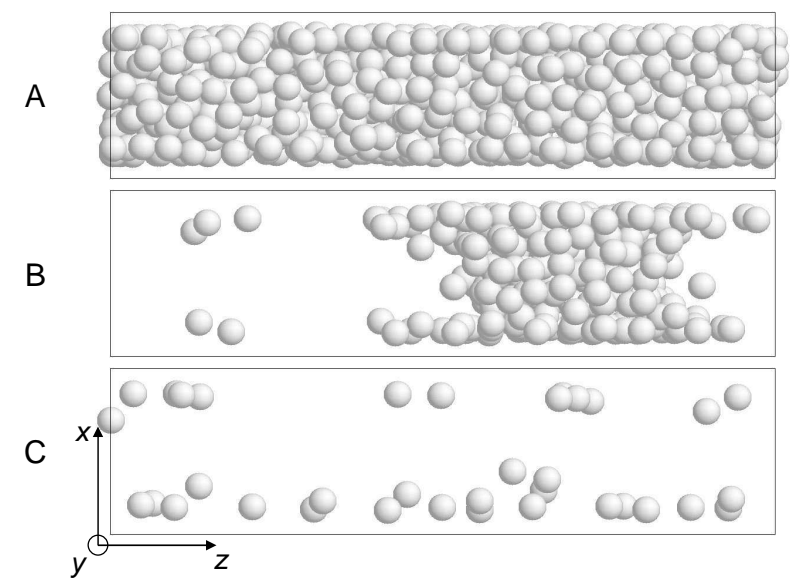

FIG. 4: Snapshots of atomic configurations for the confined fluid when the reservoir-to-pore volume ratio is $r=667$. The points $A, B$, and $C$ are indicated in Fig. 3b. A and C correspond to laterally uniform liquidlike and gaslike states, respectively, whereas point B corresponds to a nonuniform bridge-like state.

What happens if the reservoir size is further reduced? The slope of the lines of constraint given by Eq. (6) decreases and states of intermediate densities begin to appear in the isotherms. Most significantly, the system visits a branch of intermediate states that is characterized by a constant chemical potential equal to the value of liquid-gas coexistence in the pore (compare with the thin vertical line in the figure). As can be seen from the sequence of isotherms in Fig. 3, the vertical extent (i.e. in adsorbed density) of the branch increases as the reservoir-to-sample volume ratio $r$ decreases and the hysteresis phenomenon between adsorption and desorption which results from a lack of equilibration on the simulation time scale, also reduces to virtually disappear for the smallest ratios $r$. Visual inspection of the atomic configurations corresponding to the intermediate vertical branch shows that the states are nonuniform along the largest $(z)$ dimension (see Fig. 4B). They have a bridge morphology that runs along the $y$ direction (much like a rail). This is clearly different from 
the liquidlike and gaslike states observed just before and just after the jump, which are uniform in the $z$ direction (see Fig. $4 \mathrm{~A}$ and $4 \mathrm{C}$ ). Comparing the atomic configurations of the vertical branch obtained for the different ratios $r$ confirms that the same bridge (or rail) morphology is found (compare Figs. 4 and 5 for instance).

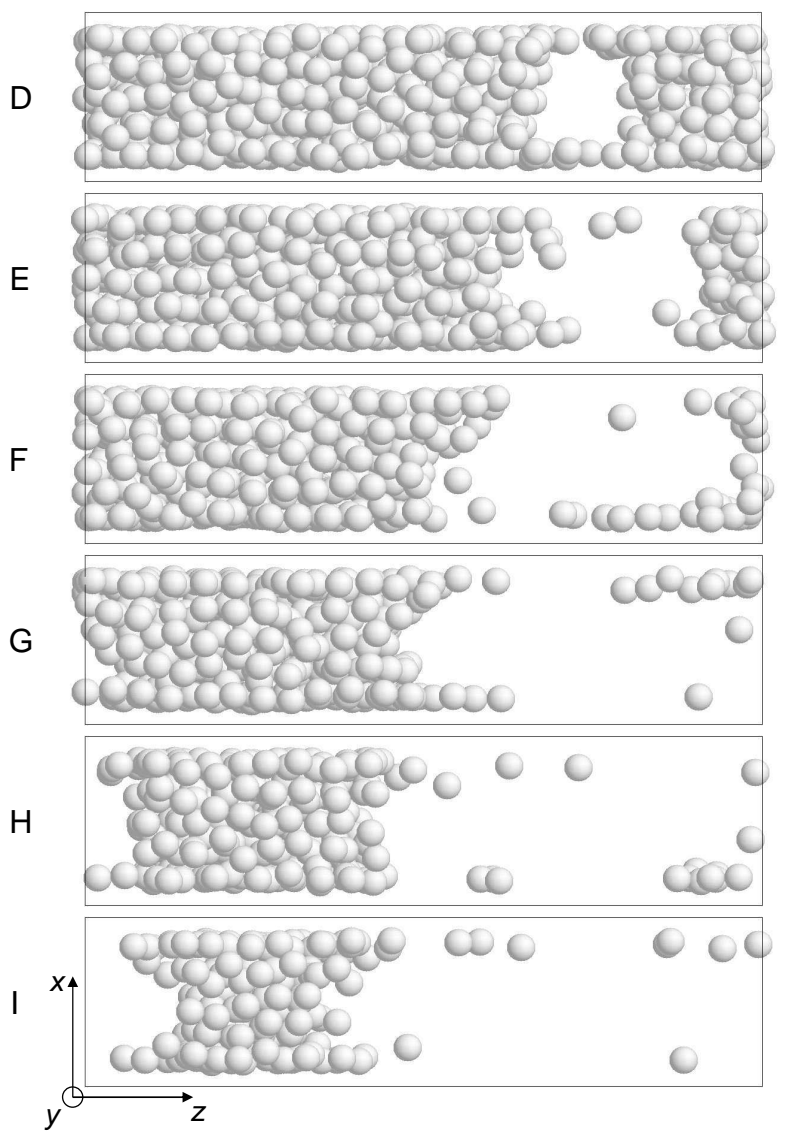

FIG. 5: Snapshots of atomic configurations for the confined fluid when the reservoir-to-pore volume ratio is $r=266$. The points $D$ to $I$ are indicated in Fig. $3 \mathrm{~d}$ and correspond (from $I$ to $D$ ) to the growth of the liquid bridge by progressive displacement of the menisci.

When the system is trapped in this branch, any variation in the total amount of particles (system plus reservoir) equals the variation in the amount adsorbed and the chemical potential remains constant. This corresponds to the displacement of the menisci on both sides of a bridge, as featured by the snapshots in Fig. 5. Most probably the menisci move without deformation since the chemical potential is constant. For the smallest reservoir-tosample ratios $(r=66.7, r=6.67$, Figs. 3e and 3f), additional intermediate states appear : either "reentrant" states near the limit of stability (spinodal) of the laterally uniform liquid- 
like and gaslike branches or states in the vicinity of the central intermediate branch but at chemical potentials different from that of "liquid-gas" coexistence. As illustrated in Fig. 6, the atomic configurations associated with the former type of states display modulations or bumps whereas those corresponding to the latter type display a bridge-like morphology (with probably in this case a deformation of the two menisci, which affects the chemical potential). As already mentioned, hysteresis is no longer observed for the adsorption/desorption isotherm studied with the smallest reservoir-to-sample ratios (see Figs. 3e and 3f). ${ }^{23}$

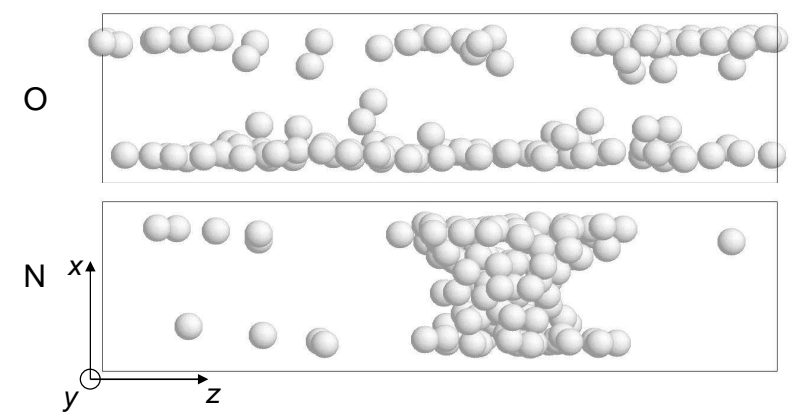

FIG. 6: Snapshots of atomic configurations for the confined fluid when the reservoir-to-pore volume ratio is $r=6.67$ (Fig. 3f). The points $O$ and $N$ are on the two sides of the discontinuity in the bottom part of the isotherm. $N$ corresponds to a bridge-like state whereas $O$ corresponds to undulations (bumps) in the adsorbed liquid layer.

However, despite the fact that the isotherm is reversible, there remains two distinct discontinuities corresponding to jumps in the "chemical potential" (which then has only the meaning of an intensive parameter conjugate to the number of particles but of course can no longer be controlled ${ }^{24}$ ). These can be considered as "mesoscopic morphological transitions" between different types of nonuniform states. ${ }^{1,2}$ Such phenomena ${ }^{25}$ are akin to the transitions seen in closed bulk systems near gas-liquid coexistence in a peculiar thermodynamic limit. $., 24,26,27$ The latter, which have also been dubbed explosion/implosion transition, ${ }^{8}$ correspond to a sudden rearrangement from a quasi-uniform phase to a nonuniform, droplet-like, one (or the reverse). In the present case, it is worth stressing that the observed discontinuities do not result from lack of equilibration. It is not that the system is trapped in metastable states due to a too small simulation length but rather that no acceptable states are present in the range of adsorbed density and chemical potential associated with the jumps. 


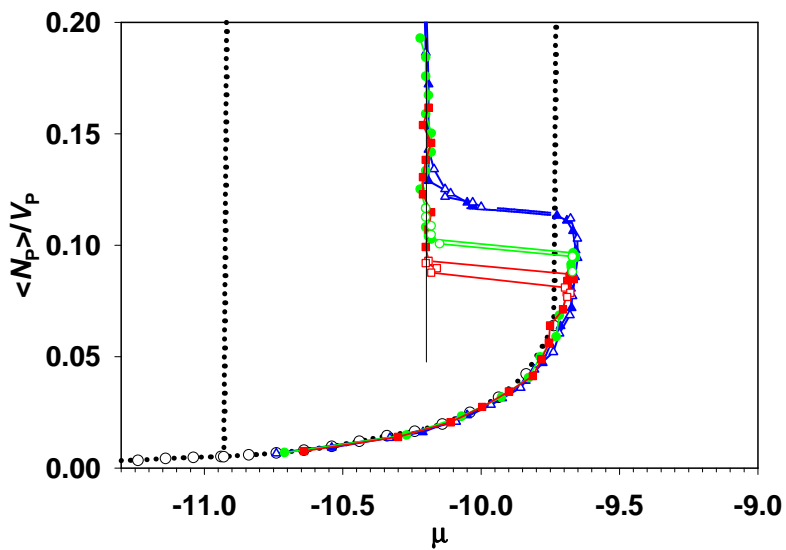

FIG. 7: System-size dependence of a "mesoscopic morphological transition". Close-up on the adsorption/desorption isotherm for a reservoir-to-sample volume ratio $r=6.67$ and 3 different pore lengths: $L=24$ (triangles), $L=32$ (circles), and $L=40$ (squares). Note that, as $L$ increases, the difference in adsorbed density $\Delta \rho$ between the intermediate branch and the lower one at $\mu \simeq-10.25$ (value at gas-liquid coexistence) decreases and hysteresis increases.

The "mesoscopic morphological transitions" depend in a nontrivial way on the system size. $^{8,24,26,27}$ To investigate this feature, we have repeated the simulation for $r=6.67$ with larger pore lengths, $L=32$ and $L=40$ (we recall that most of our simulations are done for $L=24$, see Fig. 1 and its caption) and we consider the transition in the low-density region of the isotherm. As illustrated in Fig. 7, one observes as $L$ increases that the discontinuity in $\mu$ persists and that the hysteresis between adsorption and desorption, which was absent for $L=24$, develops. Longer simulation times would now be required to make the isotherm reversible. One can also see that the difference in adsorbed density $\Delta \rho$ between the branch of bridge-like states and the lower, gas-like branch (for the value of $\mu$ corresponding to gasliquid coexistence) shrinks as $L$ becomes larger. The difference being due to a liquid "rail" of width equal to a few atomic diameters $\sigma$, the associated change in density is expected to decrease as $\sigma / L$ as $L \rightarrow+\infty$. The data in Fig. 7 do not cover a wide enough range of $L$ to precisely confirm the $1 / L$ behavior but is nonetheless compatible with it. The size of the discontinuity $\Delta \mu$ does not vary much with $L$ for the present sizes , but inspection of the shape of the lower gas-like branch in Fig. 7 indicates that for larger values of $L$, as $\Delta \rho$ gets smaller, it should start decreasing and go to zero (presumably as $1 / L$ too). We therefore conclude that as for their counterparts in closed bulk systems, the behavior of the 
"mesoscopic morphological transitions" is rather subtle in the thermodynamic limit.

\section{Stability of states and relation to capillary condensation kinetics}

An important question to be addressed is that of the nature and the origin of the stability of the various fluid states previously obtained. In the limit of an infinite time for equilibration, equilibrium is reached. Metastability (except at zero temperature or in meanfield approximations) implies a finite observation time, which can therefore be shorter than the time needed to escape from a given state. The state is then considered as metastable: it is at least mechanically stable or "locally stable" in some coarse-grained free energy or thermodynamic-like potential (whose construction can be made more rigorous ${ }^{28,29}$ ). By contrast, even at equilibrium, a state which is unstable or metastable in a grand-canonical setting (open system) can be stabilized by a constraint and therefore be considered as "conditionally stable". This phenomenon is well known in the case of nucleation droplets in a bulk fluid near gas-liquid coexistence in a canonical ensemble (constraint of fixed number of particles).

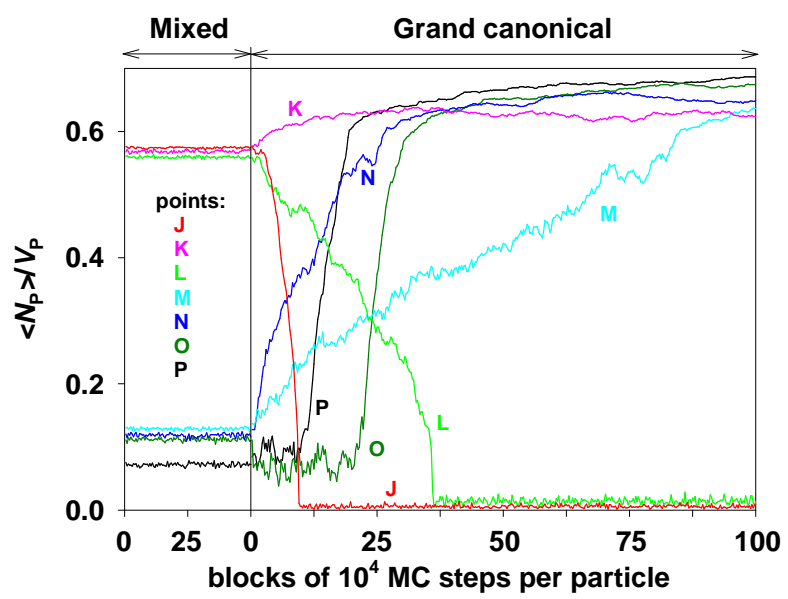

FIG. 8: Evolution of the various states labeled $J$ to $P$ for an initial reservoir-to-pore volume ratio $r=6.67$ (see Fig. 3f) in the mesoscopic canonical ensemble when switched to the grand-canonical ensemble. None of the intermediate states are "metastable" in the grand-canonical ensemble.

In our previous paper on adsorption in intrinsically inhomogeneous mesoporous media, ${ }^{7}$ we have shown that the states inside the hysteresis loop that could be reached within the mesoscopic canonical ensemble were metastable in grand canonical ensemble. We have 
performed the same analysis for the (laterally) uniform and nonuniform states found in the slit mesopore, in the case where the reservoir-to-pore volume ratio almost corresponds to canonical conditions and in which full equilibration is attained $(r=6.67)$. We proceed as follows. Starting from a given state found in the mesoscopic canonical ensemble (states labeled $J$ to $P$ in Fig. 3f), we run the usual GCMC algorithm with the value of the chemical potential found in the mesoscopic canonical ensemble. Fig. 8 shows the evolution of the amount adsorbed during the course of the simulation. As can be seen, after a transient period all states reach one of the two GCMC branches. For instance, starting from points $M, N$, $O$ and $P$ (these points are above coexistence), the system reaches the GCMC liquidlike stable state. Note that for point $O$, the system initially reaches the GCMC gaslike state, and finally jumps to the liquidlike state. For points $J$ and $L$ (below coexistence), the system reaches the GCMC gaslike stable state. However, we find that the system does not necessarily reach the stable portions of the GCMC branches (due to the finite simulation time scale), as shown by point $K$ which reaches the metastable part of the GCMC liquidlike branch. Anyhow, this demonstrates that the intermediate states are conditionally stable but not metastable. For instance, the bridge-like states observed at the chemical potential characterizing coexistence are probably saddle-points in the grand-potential landscape with one flat direction (corresponding to motion of the menisci).

A somewhat different question concerns the stability of all states obtained in the mesoscopic canonical ensemble when a hysteresis is observed. In this case, some of the states found in the portions of branches forming the hysteresis loop or loops are not stable (even conditionally) but "conditionally metastable" : the width of the loops obtained by Monte Carlo depends on the length of the simulation and is expected to disappear at true equilibrium. The corresponding equilibrium transition (occuring for equality of the total Helmholtz free energy on both sides of the discontinuity) most certainly passes somewhere in the middle of the hysteresis loop(s) (see Fig. 9). As the reservoir size decreases and the canonical situation is approached, we observe that the simulation length (which is constant in our study) becomes sufficient for equilibration and the isotherm becomes reversible. This indicates that the free energy barriers that trap the adsorbed fluid in "conditionally metastable" states decrease with the relative size of the reservoir. Not surprisingly, the nucleation barrier opposing the passage from a gaslike configuration to a liquidlike one in an open system is significantly higher than the barrier which is on the way to the transformation from a 


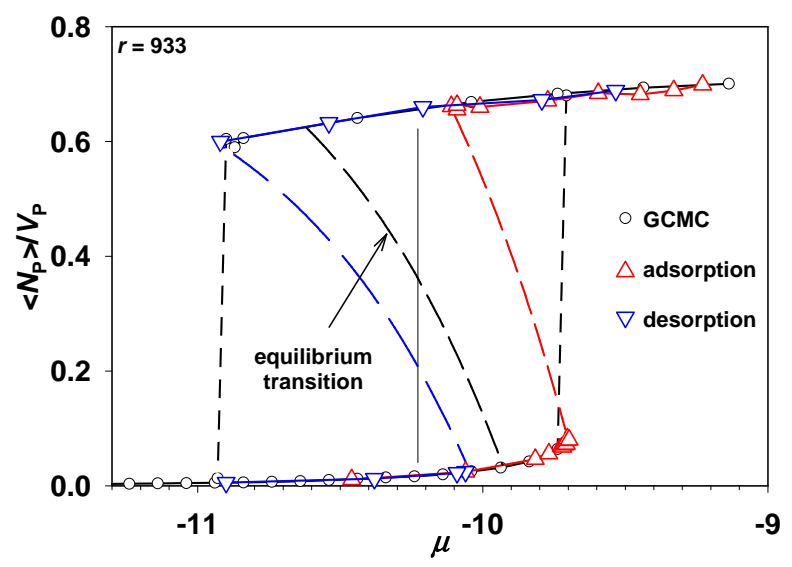

FIG. 9: Adsorption/desorption isotherms for a Lennard-Jones fluid in a slit pore obtained within the mesoscopic canonical ensemble for a reservoir-to-pore volume ratio $r=933$ (same data as in Fig. 3a). The dashed lines follow the constraint relation in Eq. (6). The intermediate curve corresponds to the equilibrium transition with equality of the total Helmholtz free energy on both sides of the discontinuity.

gaslike state to a bridge-like state (or from a liquidlike state to a bridge-like state) in a closed system.

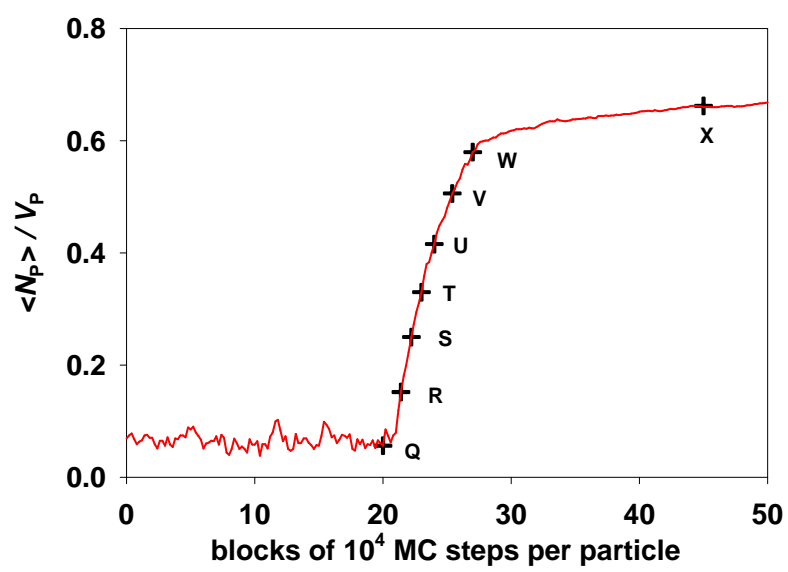

FIG. 10: GCMC capillary condensation corresponding to the jump in the adsorption isotherm in Fig. 2. Evolution with the number of simulation steps of the adsorbed fluid density.

Finally, we have compared the sequence of conditionally stable states appearing in the mesoscopic canonical ensemble (Figs. 3d and 3f) with the states visited in the GCMC "dynamics" by the fluid in a grand-canonical setting during the out-of-equilibrium capillary condensation (Fig. 2). Although the GCMC "dynamics" is not directly related to the 


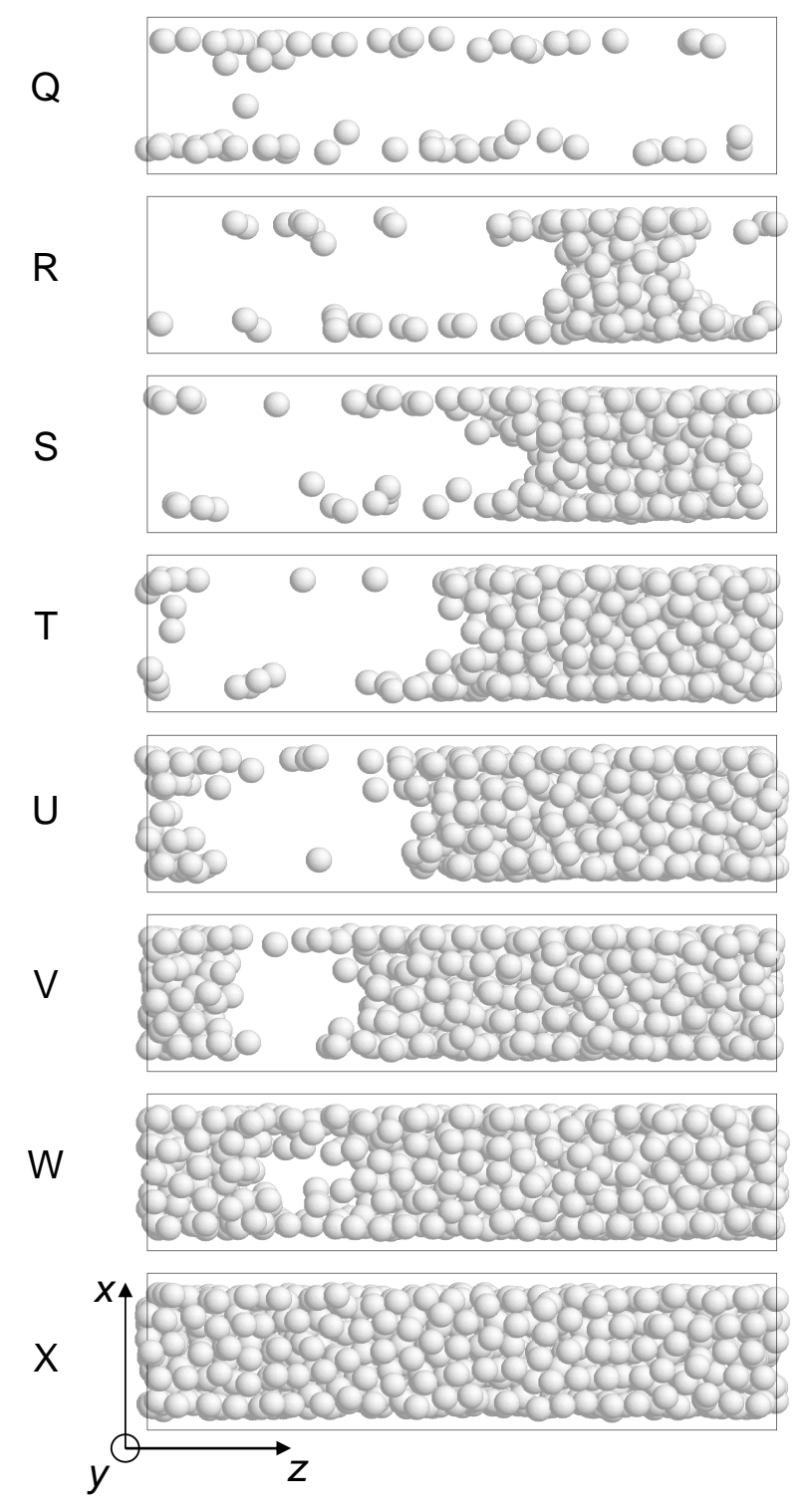

FIG. 11: GCMC capillary condensation corresponding to the jump in the adsorption isotherm in Fig. 2. Sequence of snapshots of atomic configurations for the confined fluid during condensation for the points labeled in 10 .

true motion of the atoms, it is nonetheless expected to provide, when no fancy acceleration algorithms are used, an indication on the states through which a system actually passes. As a function of GCMC "time" (i.e., number of steps), the adsorbed amount of fluid increases as shown in Fig. 10 and the corresponding sequence of representative atomic configurations is displayed in Fig. 11. One can see that bumps first appear in the adsorbed layers, followed by the occurence of a liquid bridge (a "rail") that grows by displacement of its two menisci 
until only a gas bubble (with a cylindrical morphology) remains. Complete filling takes place after the disappearance of this bubble. The sequence is strikingly similar to that found at equilibrium in the mesoscopic canonical ensemble (Figs. 5 and 6), which is in agreement with the Everett-Haynes scenario for capillary condensation in pores of ideal geometry. ${ }^{1}$ Note however that the condensation phenomenon that we observe in the GCMC simulation is out of equilibrium and associated with hysteresis. A similar conclusion has been recently reached by Monson on the basis of a mean-field kinetic theory. ${ }^{6}$

\section{CONCLUSION}

By studying the adsorption of a fluid in a slit pore, we have followed the progressive appearance, as the reservoir-to-pore volume ratio decreases to eventually reach canonical ensemble conditions, of states of intermediate density between the gaslike and liquidlike branches that are found in a grand-canonical setting. The intermediate states are nonuniform, i.e. they break the symmetry of the slit pore geometry in one direction, taking the morphology of liquid bridges (or "rails"), gas bubbles (or gas "cylinders") and of undulations or bumps of the liquid layers. These states are "conditionally stable", being stabilized by the constraint of fixed total number of particles in the pore-plus-reservoir system. However, we have shown that they are not "metastable" under grand-canonical conditions. This is at odds with the intermediate states found in an intrinsically inhomogeneous porous medium. ${ }^{7}$

Note that in this study we have only found configurations that (spontaneously) break the translational symmetry in one direction. The size of the simulation cell in the other direction parallel to the pore walls is probably too small to accomodate configurations that are also nonuniform in this direction. As a consequence, we cannot describe the nucleation barriers for capillary condensation in an infinite slit pore (in a grand-canonical setting): for instance, the observed bridge-like (or bump-like) states, which represent saddle points in the grand-canonical ensemble that are stabilized by the (quasi) canonical constraint, are not the proper candidates for activated states as they are infinite in one direction and consequently correspond to an infinite (i.e., system-size dependent) free-energy barrier. The influence of the system size (i.e., the size of the simulation cell comprising the pore) is presently under investigation.

Interestingly, we have observed that the isotherms become more and more reversible, and 
therefore closer to equilibrium condition, when we diminish the relative size of the reservoir at constant total simulation length and constant sample size. This indicates that metastability in the canonical ensemble is associated with shorter time scales than in the grand-canonical ensemble. In the (quasi) canonical situation, we have also shown that discontinuities can still be found in the isotherm, which correspond to (system-size dependent) "mesoscopic morphological transitions" between different types of intermediate states.

* Electronic address: puibasset@cnrs-orleans.fr

$\dagger$ Electronic address: edouard.kierlik@upmc.fr

$\ddagger$ Electronic address: tarjus@lptl.jussieu.fr

1 D. H. Everett and J. M. Hayes, J. Colloid and Interface Sci. 38, 125 (1972).

2 K. G. Kornev, I. K. Shingareva, and A. V. Neimark, Adv. Colloid Interface Sci. 96, 143 (2002).

3 A. V. Neimark, P. I. Ravikovitch, and A. Vishnyakov, Phys. Rev. E 65, 031505 (2002).

4 A. Vishnyakov and A. V. Neimark, J. Chem. Phys. 119, 9755 (2003).

5 E. A. Ustinov and D. D. Do, J. Phys. Chem. B 109, 11653 (2005).

6 P. A. Monson, J. Chem. Phys. 128, 084701 (2008).

7 E. Kierlik, J. Puibasset, and G. Tarjus, J. Phys.: Condens. Matter 21, 155102 (2009).

8 M. K.-H. Kiessling and J. K. Percus, J. Stat. Phys. 78, 1337 (1995).

9 Bonnet F, Lambert T, Cross B, Guyon L, Despetis F, Puech L and Wolf PE 2008 Eur. Phys. Lett.82 6003.

10 A. V. Neimark and A. Vishnyakov, Phys. Rev. E 62, 4611 (2000).

11 A. V. Neimark and A. Vishnyakov, J. Phys. Chem. B 109, 5962 (2005).

12 A. V. Neimark and A. Vishnyakov, J. Chem. Phys. 122, 054707 (2005).

13 A. V. Neimark and A. Vishnyakov, J. Chem. Phys. 122, 234108 (2005).

14 A. Z. Panagiotopoulos, Mol. Phys. 61, 813 (1987).

15 J. Puibasset, J. Phys. Chem. B 109, 4700 (2005).

16 J. Puibasset, J. Chem. Phys. 122, 134710 (2005).

17 B. Smit, P. De Smedt, and D. Frenkel, Mol. Phys. 68, 931 (1989).

18 B. Smit and D. Frenkel, Mol. Phys. 68, 951 (1989).

19 B. Widom, J. Chem. Phys. 39, 2808 (1963). 
20 J. S. Rowlinson and B. Widom, Molecular Theory of Capillarity (Clarendon Press, Oxford, 1982).

21 R. Eppenga and D. Frenkel, Mol. Phys. 52, 1303 (1984).

22 V. I. Harismiadis, J. Vorholz, and A. Z. Panagiotopoulos, J. Chem. Phys. 105, 8469 (1996).

23 The accuracy of the calculation was improved and checked by decreasing the increment in the total amount of particles: we find that the results are unchanged for increments below \pm 5 atoms (the hysteresis shown in Fig. 3e is for increments of \pm 2 atoms).

24 M. Biskup, L. Chayes, and R. Kotecký, Physica A 327, 583 (2003).

25 For a technical reason, the purely canonical case could not be considered with our algorithm since the atoms are introduced in the reservoir. However, considering the fact that for a ratio 6.67, the number of particles in the reservoir during the transition between the liquidlike and inhomogeneous states (respectively gaslike and inhomogeneous states) is, on average, at the most 3 (respectively 11) while the number of particles in the system is around 650 (respectively 130), the isotherm is probably very close to the canonical limit. In this case, fluctuations of the number of particles in the reservoir may be large but the effect on the value of the chemical potential remains small. See ${ }^{13}$ for a more detailed discussion.

26 M. Biskup, L. Chayes, and R. Kotecký, Europhys. Lett. 60, 21 (2002).

27 K. Binder, Physica A 319, 99 (2003).

28 B. Gaveau and L. S. Schulman, J. Math. Phys. 39, 1517 (1998).

29 G. Biroli and J. Kurchan, Phys. Rev. E 64, 016101 (2001).

30 D. Frenkel and B. Smit, Understanding Molecular Simulation (Academic Press, London, 1996).

\section{Appendix: Thermodynamics in the mesoscopic canonical ensemble}

The system being the reunion of two subsystems (pore and reservoir), its partition function can be written as the Gibbs partition function ${ }^{17,30}$ restricted to fixed volumes:

$$
\begin{aligned}
Z\left(N, V_{P}, V_{R}, T\right) & =\sum_{N_{P}=0}^{N} \frac{V_{R}^{N-N_{P}}}{\Lambda^{3\left(N-N_{P}\right)}\left(N-N_{P}\right) !} \\
& \times \frac{1}{\Lambda^{3 N_{P} N_{P} !}} \int_{V_{P}} d \mathbf{r}_{1} . . d \mathbf{r}_{N_{P}} \exp \left[-\beta U_{P}\left(\mathbf{r}_{1}, . ., \mathbf{r}_{N_{P}}\right)\right]
\end{aligned}
$$

where $U_{P}$ is the configurational energy of $N_{P}$ fluid atoms inside the pore and the fluid is supposed to be ideal in the reservoir. This partition function is equal to the canonical one $Z\left(N, V=V_{P}+V_{R}, T\right)$ for the whole pore-plus-reservoir system. The Helmholtz free energy is $F=-k T \ln Z$ and the chemical potential $\mu$ for the whole pore-plus-reservoir system is 
defined in Eq. (1) or, more precisely, at a discrete atomic level, as $\mu=-k T \ln [Z(N+$ $\left.\left.1, V_{P}, V_{R}, T\right) / Z\left(N, V_{P}, V_{R}, T\right)\right]$.

We stress that the chemical potential $\mu$ can be a discontinuous function of $N$ at constant $V_{P}, V_{R}, T$ (which makes its denomination as a "chemical potential" awkward in such cases ${ }^{24}$ ). The Helmholtz free energy $F$ of the whole system is however always continuous, which allows one to keep using thermodynamic integration for its computation (see section IV).

In order to show the equality of the chemical potentials $\widetilde{\mu}_{R}, \widetilde{\mu}_{P}$ defined in Eqs. $(3,4)$, we calculate the quantity:

$$
\begin{aligned}
& \left\langle\frac{1}{V_{P}} \int_{V_{P}} d \mathbf{r} e^{-\beta W_{i n s}(\mathbf{r})}\right\rangle \\
& =\frac{1}{V_{P} Z_{N}} \sum_{N_{P}=0}^{N} \frac{V_{R}^{N-N_{P}}}{\Lambda^{3\left(N-N_{P}\right)}\left(N-N_{P}\right) !} \frac{1}{\Lambda^{3 N_{P}} N_{P} !} \\
& \times \int_{V_{P}} d \mathbf{r}_{1} . . d \mathbf{r}_{N_{P}+1} \exp \left[-\beta U_{P}\left(\mathbf{r}_{1}, . ., \mathbf{r}_{N_{P}}\right)+W_{i n s}\left(\mathbf{r}_{N_{P}+1}\right)\right] \\
& =\frac{\Lambda^{3}}{V_{P} Z_{N}} \sum_{N_{P}=1}^{N+1} N_{P} \frac{V_{R}^{N+1-N_{P}}}{\Lambda^{3\left(N+1-N_{P}\right)}\left(N+1-N_{P}\right) !} \\
& \times \frac{1}{\Lambda^{3 N_{P} N_{P} !}} \int_{V_{P}} d \mathbf{r}_{1} . . d \mathbf{r}_{N_{P}} \exp \left[-\beta U_{P}\left(\mathbf{r}_{1}, . ., \mathbf{r}_{N_{P}}\right)\right]
\end{aligned}
$$

where we have redefined the variable in the sum as: $N_{P}+1 \rightarrow N_{P}$. The above expression can be rewritten

$$
\left\langle\frac{1}{V_{P}} \int_{V_{P}} d \mathbf{r} e^{-\beta W_{i n s}(\mathbf{r})}\right\rangle=\frac{\Lambda^{3}}{V_{P} Z_{N}} Z_{N+1}\left\langle N_{P}\right\rangle_{N+1},
$$

where $\left\langle N_{P}\right\rangle_{N+1}$ is the average number of particles in the pore when the total number of particles in the system is equal to $N+1$. By using the above definition of the chemical potential $\mu$ and neglecting the difference between $\left\langle N_{P}\right\rangle_{N+1}$ and $\left\langle N_{P}\right\rangle_{N}$ (which is valid in the large $N$ limit except at the location of a discontinuity proportional to $V_{P}$ that occurs in $\left\langle N_{P}\right\rangle$ when passing from $N$ to $N+1$ ), it finally follows that

$$
\left\langle\frac{1}{V_{P}} \int_{V_{P}} d \mathbf{r} e^{-\beta W_{i n s}(\mathbf{r})}\right\rangle=e^{-\beta \mu} \frac{\left\langle N_{P}\right\rangle_{N} \Lambda^{3}}{V_{P}} .
$$

As a consequence, the chemical potential of the fluid in the pore $\widetilde{\mu}_{P}$ as defined by Eq. (4) is equal to $\mu$. The same property holds for the fluid in the reservoir (provided that the latter is large enough to neglect the fluctuations in the number of particles): $\widetilde{\mu}_{R}=\mu$.

This equality between the chemical potentials, $\widetilde{\mu}_{P}=\widetilde{\mu}_{R}$, which in turn are both equal to the true chemical potential $\mu$, constitutes a criterion for the convergence of the simulation. 

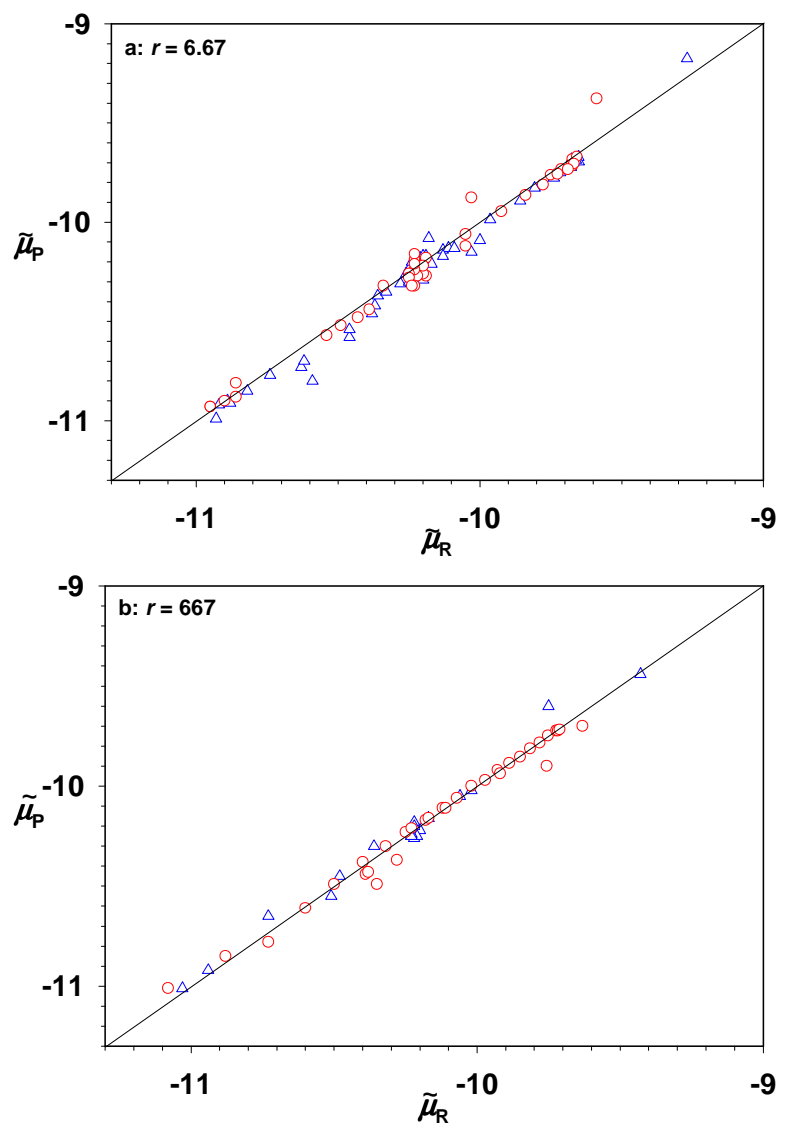

FIG. 12: Comparison of the chemical potentials defined for the Lennard-Jones fluid in the pore $\widetilde{\mu}_{P}$ and in the reservoir $\widetilde{\mu}_{R}$ after equilibration for two different ratios of the reservoir-to-pore volumes $r$.

In Fig. 12, $\widetilde{\mu}_{P}$ is plotted against $\widetilde{\mu}_{R}$ for two volume ratios $r$ between the reservoir and the pore volumes. As can be seen, the points are aligned along the first diagonal, except for the highest chemical potential values that correspond to dense states for which the insertion method is expected to converge very slowly. ${ }^{13}$ Note that when discontinuities are present, the branches of $\widetilde{\mu}_{P}, \widetilde{\mu}_{R}$ and $\mu$ on either sides of the discontinuities should still coincide at chemical equilibrium. 